\title{
On the Performance of NCC-OFDMA with Single Relay Selection
}

\author{
Semiha Tedik Başaran and Güneş Karabulut Kurt \\ Department of Electronics and Communication Engineering \\ Istanbul Technical University \\ Email: \{tedik, gkurt\}@itu.edu.tr
}

\author{
Ioannis Chatzigeorgiou \\ School of Computing and Communications \\ InfoLab21, Lancaster University \\ Email: i.chatzigeorgiou@lancaster.ac.uk
}

\begin{abstract}
In this paper, we investigate the outage probability of a network coded cooperation (NCC) system in the presence of frequency selective channels with the usage of orthogonal frequency division multiple access (OFDMA) technique. As OFDMA offers a flexible design of bandwidth usage by letting smart subcarrier allocation schemes in the frequency selective channels, combining NCC with OFDMA leads to feasible transmission schemes for efficient resource utilization. A single relay selection (SRS) technique is used to alleviate the complexity of utilizing all relay nodes. This system model is referred to as NCC-OFDMA-SRS. The first approximation of outage probability expressions of the NCC-OFDMA-SRS system is derived, and the results are supported by extensive simulations. In addition, asymptotic outage probability expressions are obtained, showing that the achievable maximum diversity gain results are attained.
\end{abstract}

Index Terms-Dense network, network coded cooperation, network coding, OFDMA, relay selection.

\section{INTRODUCTION}

Network coding is a promising technique which allows cross-layer optimization by providing robustness against the error propagation and an increase in throughput for wireless networks [1]. Relay nodes use network coding coefficients, which are selected from a field of size $q$ denoted by $\mathcal{G F}(q)$, to create network-coded symbols. Each detected source symbol is multiplied with the corresponding network code coefficient and all terms are summed. In wireless networks, a direct link may exist between the source and the destination due to the propagation characteristics of the wireless channel in each direction of a network coded system. This system, named as network coded cooperation (NCC) system, is proposed in [2].

The simultaneous use of all relay nodes may create trouble about efficient use of bandwidth in NCC systems due to the feedback requirements about the channel states [3]. NCC systems require exact channel estimations of all relay to destination links to obtain source symbols. Hence, a relay selection scheme is important for simplification of the NCC system implementation. Selecting the best relay node instead of using all relays gives the full diversity gain (equal to one greater than the number of relay nodes) for the cooperative communication. Surprisingly, as shown in [4], [5], these results are not valid for NCC systems. If the number of selected relays is less than the number of sources, the full diversity gain cannot be achieved and the diversity order is equal to one greater than the number of selected relays [4]. Hence, it is demonstrated that the diversity order of an NCC system with single relay selection can reach up to 2 without using any multiplexing scheme. Multiple relay selection cases for NCC systems are also investigated in [5].

Orthogonal frequency division multiple access (OFDMA) is the most frequently used multiplexing technique in wireless communications. The OFDMA extension of NCC abbreviated by NCC-OFDMA is also investigated as a resource scaling technique in NCC systems [6]. On the other hand, the diversity gain and decoding probability analysis of relay selection of NCC-OFDMA system is not yet available in the literature. In this paper, the decoding probability of an NCC-OFDMA system with a single relay selection indicated by NCC-OFDMA-SRS, is investigated. The expression of diversity order of NCC-OFDMA-SRS system in the asymptotic SNR region is derived. Maximum distance separable (MDS) codes are used at the relay nodes, and the Random Rotation and Expansion based Hopcroft-Karp $\left(\mathrm{R}^{2} \mathrm{EHK}\right)$ algorithm [7] is implemented in the subcarrier allocation part in both of the transmission phases with $L$ coherence bandwidths. Min-max relay selection criteria are used to determine the best SNR in a set that contains the worst SNR of each pair of source-to-relay (sr) and relay-to-destination (rd) links. We establish that the diversity order of NCC-OFDMA-SRS depends on $L$ and is equal to $2 L$. The accuracy of the theoretical findings is supported by extensive simulation results.

\section{System ModeL}

The NCC-OFDMA system contains $P$ source nodes, $M$ relay nodes, and one destination node. The total number of subcarriers is denoted by $N$, satisfying the condition $N>\{P, M\}$. This assumption is realistic since the number of subcarriers is usually greater than the number of source and relay nodes. The number of coherence bandwidths of the NCC-OFDMA system, indicated by $L$, could be smaller than $N$ if the channel is frequency selective. The transmission is completed in two orthogonal phases, namely the broadcast phase and the relaying phase. In the broadcast phase, source nodes radiate their symbols through the wireless channel. Due to the broadcast nature of the wireless channel, the 
symbols of the source nodes can be overheard by the relay nodes and the destination node.

Let the frequency domain representation of the $n^{\text {th }}$ subcarrier channel gain of $u$ be given by $H_{u}[n]$, where $u \in\left\{\mathrm{s}_{i} \mathrm{~d}, \mathrm{~s}_{i} \mathrm{r}_{j}, \mathrm{r}_{j} \mathrm{~d}\right\}, i=1, \ldots, P, j=1, \ldots, M, n=$ $1, \ldots, N$. It is assumed that $H_{u}[n]$ has Rayleigh distribution. The received symbols at the $j^{\text {th }}$ relay node and destination node in the broadcast phase are given respectively, as:

$$
\begin{aligned}
Y_{\mathrm{s}_{i} \mathrm{r}_{j}}[n] & =H_{\mathrm{s}_{i} \mathrm{r}_{j}}[n] X_{i}[n]+W_{\mathrm{s}_{i} \mathrm{r}_{j}}[n] \\
Y_{\mathrm{s}_{i} \mathrm{~d}}[n] & =H_{\mathrm{s}_{i} \mathrm{~d}}[n] X_{i}[n]+W_{\mathrm{s}_{i} \mathrm{~d}}[n],
\end{aligned}
$$

where each symbol $X_{i}[n]$ is transmitted at unit power. The additive white Gaussian noise (AWGN) at the $j^{\text {th }}$ relay node and the destination node in the broadcast phase are denoted by $W_{\mathrm{s}_{i} \mathrm{r}_{j}}[n]$ and $W_{\mathrm{s}_{i} \mathrm{~d}}[n]$, respectively. The $j^{\text {th }}$ relay node detects $X_{i}[n]$ by using $Y_{\mathrm{s}_{i} \mathrm{r}_{j}}[n]$. The detected version of $X_{i}[n]$ is represented by $\tilde{X}_{i}[n]$. The SNR values of sr links are assumed to be higher than those of source-to-destination (sd) links. Hence, the subcarrier assignment is carried out based on the qualities of the sd links.

Then, the selected relay node generates the network coded symbol by first multiplying each $X_{i}[n]$ by a network coefficient $\alpha_{i j}$ and then adding the $P$ products. The coefficients are selected either from an MDS code satisfying the Singleton bound or randomly selected from $\mathcal{G} \mathcal{F}(q)$ as in RLNC with sufficiently large $q$. The network coded symbol of the $j^{\text {th }}$ relay node can be calculated as:

$$
C_{j}[n]=\alpha_{1 j} \tilde{X}_{1}[n]+\alpha_{2 j} \tilde{X}_{2}[n] \ldots,+\alpha_{P j} \tilde{X}_{P}[n],
$$

where + represents the summation operator in $\mathcal{G F}(q)$. The received symbol at the destination node in the relaying phase equals to:

$$
Y_{\mathrm{r}_{j} \mathrm{~d}}[n]=H_{\mathrm{r}_{j} \mathrm{~d}}[n] C_{j}[n]+W_{\mathrm{r}_{j} \mathrm{~d}}[n],
$$

where $W_{\mathrm{r}_{j} \mathrm{~d}}[n]$ indicates the AWGN component. Hence, the destination node has multiple symbols.

The transmission is realized by OFDMA in both phases. The subcarrier selection process is critical in order to enhance the NCC-OFDMA system performance. The bipartite graph model is preferred for the assignment of subcarriers to the users [7] by introducing a maximum matching problem. The Hopcroft-Karp algorithm gives the maximum cardinality matching for bipartite graphs [8]. Hence, the minimum number of users in outage is calculated by using the Hopcroft-Karp algorithm in the subcarrier assignment problem. The $\mathrm{R}^{2} \mathrm{EHK}$ algorithm, which provides the fairness between all nodes [7], is an extended version of the Hopcroft-Karp algorithm. In $\mathrm{R}^{2} \mathrm{EHK}$ algorithm, the vertices are randomly rotated with equal probability to guarantee the fairness among users. In addition, if the number of users is lower than the number of subcarriers, the vertices are expanded based on the required number of subcarriers of each vertex. It is used to allocate the subcarriers [7] and to achieve the optimal outage probability. It exploits both frequency and multiuser diversity and gives the minimum outage probability in OFDMA systems, while giving the diversity order of $L$ under the condition of $L<P \leq N$ [7]. Hence, each link has $L$ independent transmission channels. In the relaying phase, a single relay is chosen according to $H_{\mathrm{s}_{i} \mathrm{r}_{j}}[n]$ and $H_{\mathrm{r}_{j} \mathrm{~d}}[n]$ values, as detailed in the next section.

\section{Decoding Failure Probability Analysis}

Before calculating the decoding failure probability of NCC-OFDMA-SRS, it is necessary to calculate the outage probability per link. The outage probability of transmission link $u^{l}$ in the system can be determined as:

$$
\phi_{u}^{l}=\operatorname{Pr}\left\{\log \left\{1+\left|H_{u}^{l}[n]\right|^{2} \bar{\gamma}_{u}^{l}\right\}<\mathcal{R}\right\},
$$

for $l=1, \ldots, L$, where $\bar{\gamma}_{u}^{l}$ and $\mathcal{R}$ denote the average SNR of link $u^{l}$ and target transmission rate of the system, respectively. The instantaneous SNR of link $u^{l}$ is equal to $\gamma_{u}^{l}=\left|H_{u}^{l}[n]\right|^{2} \bar{\gamma}_{u}^{l}$. And then, $\phi_{u}^{l}$ can be calculated as:

$$
\phi_{u}^{l}=\operatorname{Pr}\left\{\left|H_{u}^{l}[n]\right|^{2}<\gamma_{t h} / \bar{\gamma}_{u}^{l}\right\},
$$

where $\gamma_{t h}=2^{\mathcal{R}}-1$. If all transmission links are assumed to have Rayleigh distribution in the system, the expression in (5) becomes $\phi_{u}^{l}=1-\exp \left(-\gamma_{t h} / \bar{\gamma}_{u}^{l}\right)$. The overall decoding probability can be derived if the outage probability of each link is first calculated.

\section{A. Min-max Single Relay Selection Rule}

The optimum relay selection rule for network coding systems is related with the selection of the best relay for each source node [9]. However, this selection rule brings the inefficient use of limited bandwidth. There is another relay selection technique called min-max relay selection, which aims at maximizing the worst end-toend (source-to-relay-to-destination) SNR. Hence, minmax relay selection criteria are preferred in single-relay selection schemes to maximize the decoding probability of NCC.

Before detailing the steps of min-max relay selection, the conceptual variables will be defined. The performance of a source-to-relay-to-destination link is dominated by the weakest SNR link. Hence, the reliability of a relay node is determined by the worst SNR of all links that are connected to the relay node. The weakest SNR value of $j^{\text {th }}$ relay node can be determined through the following expression:

$$
\gamma_{j}=\min \left\{\ldots, \gamma_{\mathrm{s}_{i} \mathrm{r}_{j}}^{l}, \ldots, \gamma_{\mathrm{r}_{j} \mathrm{~d}}^{1}, \ldots, \gamma_{\mathrm{r}_{j} \mathrm{~d}}^{L}\right\},
$$

which considers coherence bandwidths. Hence, there are $(P+1) L$ different independent SNR values for each relay node. Through the independent terms in (6), the outage probability of the $j^{\text {th }}$ relay is given by:

$$
\phi_{\gamma_{j}}=1-\exp \left(-\gamma_{\mathrm{th}} / \bar{\gamma}_{j}\right)
$$




$$
\begin{aligned}
& P_{f}=1-\left[\operatorname{Pr}\left\{P \text { sd links }>\gamma_{t h}\right\}+\operatorname{Pr}\left\{P-1 \text { sd links }>\gamma_{t h}, \gamma_{\text {srs }}>\gamma_{\text {th }}\right\}\right] \\
& =\operatorname{Pr}\left\{0 \text { sd link }>\gamma_{\text {th }}\right\}+\ldots+\operatorname{Pr}\left\{P-2 \text { sd links }>\gamma_{t h}\right\}+\operatorname{Pr}\left\{P-1 \text { sd links }>\gamma_{t h}, \gamma_{\text {srs }}<\gamma_{t h}\right\} \\
& \approx\left(\prod_{i=1}^{P} \phi_{\gamma_{s_{i} d}}^{L}+\sum_{i=1}^{P}\left(\left(1-\phi_{\gamma_{s_{i} d}}^{L}\right) \prod_{\substack{i^{\prime}=1 \\
i^{\prime} \neq i}}^{P} \phi_{\gamma_{s^{\prime} d}}^{L}\right)+\ldots+\sum_{\substack{i_{1}=1, \ldots, i_{P-2}=1 \\
i_{1} \neq \ldots \neq i_{P-2} \\
\mathcal{S}=\left\{i_{1}, \ldots, i_{P-2}\right\}}}^{P}\left(\prod_{\forall i \in \mathcal{S}}\left(1-\phi_{\gamma_{s_{i} d}}^{L}\right) \prod_{\substack{i^{\prime}=1 \\
i^{\prime} \notin \mathcal{S}}}^{P} \phi_{\gamma_{s_{i^{\prime} d}}}^{L}\right)\right. \\
& \left.+\sum_{\substack{i_{1}=1, \ldots, i_{P-1}=1 \\
i_{1} \neq \ldots \neq i_{P-1} \\
\mathcal{S}=\left\{i_{1}, \ldots, i_{P-1}\right\}}}^{P}\left(\prod_{\forall i \in \mathcal{S}}\left(1-\phi_{\gamma_{s_{i} d}}^{L}\right) \prod_{\substack{i^{\prime}=1 \\
i^{\prime} \notin \mathcal{S}}}^{P} \phi_{\gamma_{i^{\prime} d}}^{L}\right) \phi_{\mathrm{srs}}^{L}\right) / P \\
& =\left(\sum_{k=0}^{P-2}\left(\sum_{\substack{i_{1}=1, \ldots, i_{k}=1 \\
i_{1} \neq \ldots \neq \ldots}}^{P} \prod_{\forall i \in \mathcal{S}}\left(1-\phi_{s_{i} d}^{L}\right) \prod_{\substack{i^{\prime}=1 \\
i^{\prime} \notin \mathcal{S}}}^{P} \phi_{\gamma_{i^{\prime} d}}^{L}\right)+\sum_{\substack{i_{1}=1, \ldots, i_{P-1}=1 \\
i_{1} \neq \ldots \neq i_{P-1}}}^{P}\left(\prod_{\forall i \in \mathcal{S}}\left(1-\phi_{\gamma_{s_{i} d}}^{L}\right) \prod_{\substack{i^{\prime}=1 \\
i^{\prime} \notin \mathcal{S}}}^{P} \phi_{\gamma_{s_{i^{\prime} d}}}^{L}\right) \phi_{\mathrm{srs}}^{L}\right) / P
\end{aligned}
$$

where $\bar{\gamma}_{j}$ represents the average value of $\gamma_{j}$ and can be computed as [9]:

$$
\bar{\gamma}_{j}=\left[\sum_{l=1}^{L}\left(\sum_{i=1}^{P} \frac{1}{\bar{\gamma}_{\mathrm{s}_{i} \mathrm{r}_{j}}^{l}}+\frac{1}{\bar{\gamma}_{\mathrm{r}_{j} \mathrm{~d}}^{l}}\right)\right]^{-1} .
$$

Hence, the SNR value of the selected relay is given as:

$$
\gamma_{\text {srs }}=\max \left\{\gamma_{1}, \ldots, \gamma_{M}\right\}
$$

Due to the mutually independent terms of $\gamma_{j}$, the outage probability of single relay selection can be calculated as:

$$
\phi_{\mathrm{srs}}=\prod_{j=1}^{M} \phi_{\gamma_{j}} \text {. }
$$

\section{B. Decoding Failure Probability of NCC-OFDMA-SRS}

At the end of the two phases, the destination node has gathered distinct symbols, which can be decoded only if $P$ of them are linearly independent. Hence, at least $P$ links should be in non-outage out of the $P+1$ available links, given that only one relay has been selected. Otherwise, the destination node cannot decode the source symbols, correctly. By using the success probability, the average decoding failure probability of the NCC-OFDMA-SRS system is calculated as:

$P_{f}=1-\left[\operatorname{Pr}\left\{P\right.\right.$ links $\left.>\gamma_{\mathrm{th}}\right\}+\operatorname{Pr}\left\{P+1\right.$ links $\left.\left.>\gamma_{\mathrm{th}}\right\}\right]$.

When considering all transmission links in the system, $P_{f}$ can be calculated as:

$$
\begin{aligned}
P_{f}= & 1-\left[\operatorname{Pr}\left\{P \text { sd links }>\gamma_{\mathrm{th}}\right\}\right. \\
& \left.+\operatorname{Pr}\left\{P-1 \text { sd links }>\gamma_{\mathrm{th}}, \gamma_{\mathrm{srs}}>\gamma_{\mathrm{th}}\right\}\right] .
\end{aligned}
$$

Theorem. Consider an NCC-OFDMA system that consists of $P$ source nodes, $M$ relay nodes and one destination node. OFDMA uses $N$ subcarriers. If the single relay selection is employed, the asymptotic decoding failure probability of the NCC-OFDMA-SRS system can be approximated by:

$$
P_{f}^{a s y m} \approx \sum_{\substack{i_{1}=1, i_{2}=1, i_{1} \neq i_{2}}}^{P} \frac{\bar{\gamma}_{\mathrm{th}}^{2 L}}{\bar{\gamma}_{\mathrm{s}_{i_{1}}}^{L} \bar{\gamma}_{\mathrm{s}_{i_{2}}}^{L}} .
$$

The theorem states that the diversity gain of the NCCOFDMA-SRS system which is independent of the values of $M$ and $P$, is equal to $2 L$.

Proof. Using (11), the decoding failure probability of NCC-OFDMA-SRS system can be calculated as in (13) by using the first order approximation of the theoretical outage probability of $\mathrm{R}^{2} \mathrm{EHK}$ algorithm given in (14) of [7]. The diversity gain of a communication system is calculated as SNR $\rightarrow \infty$. In order to investigate the asymptotic performance and find the diversity gain of NCC-OFDMA-SRS system, we will focus only on the dominant terms of $P_{f}$. In the high SNR region, (13) is dominated by the first term in the summation. Hence (13) is approximately equal to:

$$
P_{f}^{a s y m} \approx \sum_{k=0}^{P-2}\left(\sum_{\substack{i_{1}=1, \ldots, i_{k}=1 \\ i_{1} \neq \ldots \neq i_{k} \\ \mathcal{S}=\left\{i_{1}, \ldots, i_{k}\right\}}}^{P} \prod_{\forall i \in \mathcal{S}}\left(1-\phi_{\mathrm{s}_{i} \mathrm{~d}}^{L}\right) \prod_{\substack{i^{\prime}=1 \\ i^{\prime} \notin \mathcal{S}}}^{P} \phi_{\gamma_{\mathrm{s}_{i^{\prime} \mathrm{d}}}}^{L}\right) .
$$

This expression is dominated by the last term of the summation and thus, can be further reduced to:

$$
P_{f}^{a s y m} \approx \sum_{\substack{i_{1}=1, \ldots, i_{P-2}=1 \\ i_{1} \neq \ldots \neq i_{P-2} \\ \mathcal{S}=\left\{i_{1}, \ldots, i_{P-2}\right\}}}^{P}\left(\prod_{\forall i \in \mathcal{S}}\left(1-\phi_{\mathrm{s}_{i} \mathrm{~d}}^{L}\right) \prod_{\substack{i^{\prime}=1 \\ i^{\prime} \notin \mathcal{S}}}^{P} \phi_{\gamma_{\mathrm{s}^{\prime} \mathrm{d}}}^{L}\right) .
$$

Since the dominant terms are kept to find the diversity gain, the expression in (15) becomes:

$$
P_{f}^{a s y m} \approx \sum_{\substack{i_{1}=1, \ldots, i_{P-2}=1 \\ i_{1} \neq \ldots \neq i_{P-2} \\ \mathcal{S}=\left\{i_{1}, \ldots, i_{P-2}\right\}}}^{P}\left(\prod_{\substack{i^{\prime}=1 \\ i^{\prime} \notin \mathcal{S}}}^{P} \phi_{\gamma_{\mathrm{s}^{\prime} \mathrm{d}}}^{L}\right) .
$$

Using the first-order Taylor approximation, $e^{-x} \approx 1-x$, expression (12) is obtained. Hence the diversity order of the NCC-OFDMA-SRS scheme is $2 L$. 


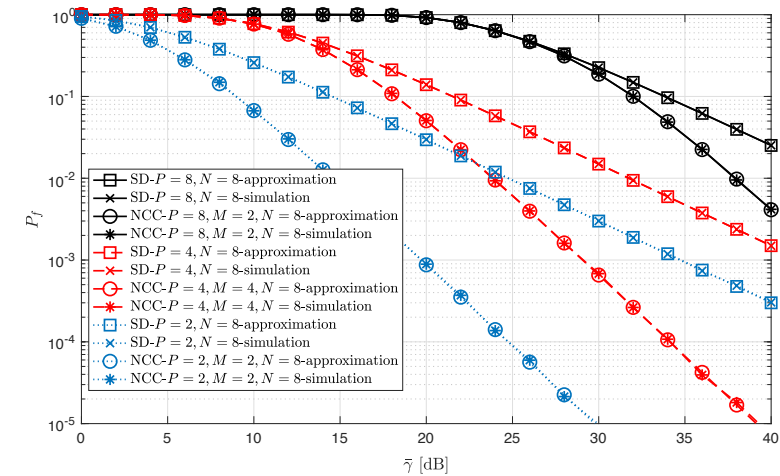

Fig. 1: Comparison of simulation and theoretical results of the decoding failure probability of NCC-OFDMA-SRS for $L=1$, $N=8$ and different values of $P$ and $M$.

\section{Simulation Results}

Comprehensive simulation results based on different system parameters that support the theoretical results are presented in this section. The results of the NCCOFDMA-SRS system are compared to the results of a system that supports only source-to-destination links, given in [7]. For brevity, we refer to the two systems as NCC and SD, respectively, in Fig. 1 and Fig. 2. It is assumed that sr links have $20 \mathrm{~dB}$ better SNR values than sd links considered to set-up relay-aided communication. The mentioned assumption is valid because relay nodes are closer to the source nodes than the destination nodes in practical applications. The $\mathrm{R}^{2} \mathrm{EHK}$ algorithm is used for assignment of the subcarriers in broadcast and relaying phases. It is assumed that only three types of links are available in the system for notational simplicity, $\bar{\gamma}_{\mathrm{s}_{i} \mathrm{~d}}=\bar{\gamma}_{\mathrm{sd}}, \bar{\gamma}_{\mathrm{s}_{\mathrm{r}_{j}}}=\bar{\gamma}_{\mathrm{sr}}$, and $\bar{\gamma}_{\mathrm{r}_{j} \mathrm{~d}}=\bar{\gamma}_{\mathrm{rd}}$. The accuracy of the theoretical derivations with the simulations is demonstrated.

The approximation and simulation results of the decoding failure probability of NCC-OFDMA-SRS system and only sd links against varying $M, P$ and $N$ values are given in Fig. 1. The number of coherence bandwidths is fixed to $L=1$ to observe the effect of other parameters. In order to increase the visualization of the effects of the system parameters, it is assumed that sd and rd links have the same average SNR value, expressed as $\bar{\gamma}=\bar{\gamma}_{s d}=\bar{\gamma}_{r d}$ and $s r$ links have the average SNR of $\bar{\gamma}_{s r}=\bar{\gamma}+20 \mathrm{~dB}$. An increase in the number of source nodes, $P$, has a negative effect on the decoding probability performance without changing the diversity gain. When only sd links are present, worse decoding performance is obtained for all cases. It is expected that the diversity order of the NCC scheme is equal to 2 when $L=1$. On the other hand, the SD system achieves a smaller diversity gain than the NCC scheme in the same conditions. Simulation results confirm the theoretical expressions.

Fig. 2 depicts the effect of the number of coherence bandwidths on system performance. It can be seen that the diversity gain of NCC-OFDMA-SRS system is only

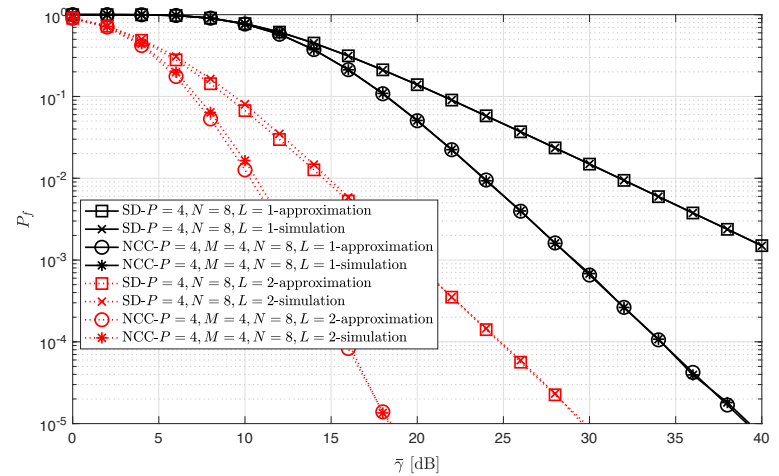

Fig. 2: Comparison of simulation and theoretical results of the decoding failure probability of NCC-OFDMA-SRS for $N=8$, $P=4, M=4$ and different values of $L$.

dependent on $L$ parameter in (12). Simulations are consistent with the theoretical results. It can be deduced from the figure that, if $L$ remains constant, the diversity order does not change. It can be observed that the SD system cannot achieve the same diversity of the NCC system.

\section{CONCLUSion}

In this paper, the performance analysis of NCCOFDMA-SRS has been examined for the first time in the literature. Analytical expressions both for the decoding failure probability and the diversity gain of NCC-OFDMA-SRS have been derived. It is proved that the diversity gain of the system is dependent only on the number of coherence bandwidths by applying the $\mathrm{R}^{2} \mathrm{EHK}$ algorithm to allocate the subcarriers. Simulation results of the decoding failure probability have verified the theoretical results.

\section{REFERENCES}

[1] R. Ahlswede, N. Cai, S.-Y. R. Li, and R. W. Yeung, "Network information flow," IEEE Trans. Inf. Theory, vol. 46, no. 4, pp. 1204-1216, July 2000.

[2] Y. Chen, S. Kishore, and J. Li, "Wireless diversity through network coding," in Proc. IEEE WCNC, Apr. 2006, pp. 1681-1686.

[3] H. Topakkaya and Z. Wang, "Wireless network code design and performance analysis using diversity-multiplexing tradeoff," IEEE Trans. Commun., vol. 59, no. 2, pp. 488-496, Feb. 2011

[4] T. X. Vu, P. Duhamel, and M. D. Renzo, "On the diversity of network-coded cooperation with decode-and-forward relay selection," IEEE Trans. Wireless Commun., vol. 14, no. 8, pp. 43694378, Aug. 2015.

[5] A. R. Heidarpour, M. Ardakani, and C. Tellambura, "Generalized relay selection for network-coded cooperation systems," IEEE Communications Letters, vol. 21, no. 12, pp. 2742-2745, 2017.

[6] Z. C. Pereira, T. H. Ton, J. L. Rebelatto, R. D. Souza, and B. F. Uchôa-Filho, "Generalized network-coded cooperation in ofdma communications," IEEE Access, vol. 6, pp. 6550-6559, 2018

[7] B. Bai, W. Chen, K. Ben Letaief, and Z. Cao, "Diversitymultiplexing tradeoff in OFDMA systems: An H-matching approach," IEEE Trans. Wireless Commun., vol. 10, no. 11, pp. 3675-3687, Nov. 2011

[8] J. E. Hopcroft and R. M. Karp, "A $\mathrm{n}^{5 / 2}$ algorithm for maximum matchings in bipartite," in Proc. Annual Symposium on Switching and Automata Theory, Oct. 1971, pp. 122-125.

[9] Y. Li, R. H. Y. Louie, and B. Vucetic, "Relay selection with network coding in two-way relay channels," IEEE Trans. Vehic. Tech., vol. 59, no. 9, pp. 4489-4499, Nov. 2010. 